\title{
The effect of the COVID-19 pandemic on bariatric surgery delivery in Edmonton, Alberta: a single-centre experience
}

\author{
Nawaf Abu-Omar, MD \\ Gabriel Marcil, MD \\ Valentin Mocanu, MD \\ Jerry T. Dang, MD \\ Noah Switzer, MD \\ Aliyah Kanji, MD \\ Daniel Birch, MD \\ Shahzeer Karmali, MD
}

Accepted April 27, 2021

\section{Correspondence to:}

N. Abu-Omar

Department of Surgery

Royal University Hospital

Room 70 Ellis Hall, 103 Hospital Drive

Saskatoon, SK, S7N 0W8

nawaf.abuomar@usask.ca

DOI: $10.1503 /$ cjs.002421

\section{SUMMARY}

Delays in the delivery of bariatric surgery in Canada in the context of the COVID-19 pandemic have not been previously explored. Understanding the potential barriers associated with these delays may help in the implementation and delivery of enhanced bariatric protocols, thereby minimizing health care system burden and improving bariatric delivery. We present the experience of a single high-volume, accredited bariatric program in Edmonton, Alberta, in 2020. Although reductions in bariatric cases occurred during lockdown months, the overall number of cases was comparable to 2019 owing to the adoption of strategies aimed at offsetting the burden of hospital resources. These strategies included optimizing patient selection, implementing bariatric Enhanced Recovery After Surgery protocols, and minimizing unnecessary postoperative investigations to allow most patients to be discharged on postoperative day 1 . We advocate to continue optimizing bariatric delivery in the face of the COVID-19 pandemic that so disproportionally affects those with severe obesity and its metabolic complications.

$\mathrm{T}$ he COVID-19 pandemic has emerged as a substantial barrier to the delivery of elective bariatric surgical procedures in Canada, further compounding the consequences of the concurrent obesity epidemic. The chronic proinflammatory physiology and impaired immune responses associated with the metabolic derangements of obesity increase the risk for acute respiratory distress syndrome, a major contributor of COVID-19-related mortality. ${ }^{1,2}$ The disproportionate effect of COVID-19 on the bariatric population has led many to question whether bariatric procedures, which are currently the most effective treatment for severe obesity, should be rationed similarly to other nonmetabolic procedures. In support of this notion, the American Society for Metabolic and Bariatric Surgery has recently suggested that bariatric procedures instead be considered "medically necessary timesensitive surgery."3

Reducing health care burden has become a priority during the pandemic, with policy changes focused on surgeries that maximize our finite Canadian hospital resources. The extent to which these changes have influenced bariatric delivery, however, is not currently known and limits our ability to develop strategies to overcome potential barriers. We present the 2020 experience of a single high-volume, accredited bariatric program in Edmonton, Alberta, and propose a few strategies that our group has used to overcome challenges associated with the COVID-19 pandemic.

During the course of the COVID-19 pandemic in Edmonton, routine and elective health care services, including bariatric surgeries, were suspended or reduced. In our centre, the number of primary bariatric surgeries performed, excluding revisional surgeries, was marginally lower in 2020 than in 2019 (275 v. 289). However, when comparing the number of operations during the pandemic elective surgery slowdown 
months (March, April, May and December 2020) to the year prior, the difference in numbers changes drastically: there was approximately a $79 \%$ reduction in the number of bariatric surgeries during the elective surgery slowdown months (Table 1 and Figure 1). Our group was able to complete a substantial number of primary bariatric surgeries during the rest of the year by dedicating elective surgical time toward bariatric patients and implementing a specific Enhanced Recovery After Surgery (ERAS) bariatric protocol. This approach, in the optimal population, can help sustain the viability and value of high-volume bariatric surgery programs during a prolonged pandemic.

In our centre, the bariatric postoperative pathway was modified to accommodate for the exponential need for available hospital beds and to reduce the strain on hospital resources. Although ERAS protocols are widely implemented in other sectors of surgery, such as colorectal surgery, its use in bariatric surgery varies. ERAS after bariatric surgery has been shown to significantly reduce the length of hospital stay without affecting the postoperative complication rate. ${ }^{4}$ The protocol used in our centre includes adequate preoperative hydration with intravenous fluids, diet advancement to clear fluids 4 hours after an uncomplicated procedure, bariatric full fluid diet the next morning, omitting the postoperative upper gastrointestinal series leak test, and
Table 1. Primary bariatric operations at the Royal Alexandra Hospital in Edmonton, 2019-2020

\begin{tabular}{|lcc|}
\hline Month & 2019 & 2020 \\
\hline January & 29 & 28 \\
\hline February & 29 & 29 \\
\hline March & 29 & $19^{*}$ \\
\hline April & 23 & $0^{*}$ \\
\hline May & 26 & $0^{*}$ \\
\hline June & 25 & 45 \\
\hline July & 8 & 35 \\
\hline August & 12 & 35 \\
\hline September & 23 & 25 \\
\hline October & 33 & 28 \\
\hline November & 28 & 29 \\
\hline December & 24 & $2^{*}$ \\
\hline Total & 289 & 275 \\
\hline *Pandemic slow-down months. & &
\end{tabular}

ensuring adequate postoperative analgesia. We routinely perform an intraoperative leak test using a standard gastroscope and perform intraoperative laparoscopic assisted transversus abdominis plane (TAP) blocks to achieve this goal. The TAP blocks help to minimize postoperative narcotic use, which in turn reduces nausea and vomiting. Implementing these standardized measures resulted in discharging more than $80 \%$ of patients on postoperative day 1 after an uncomplicated

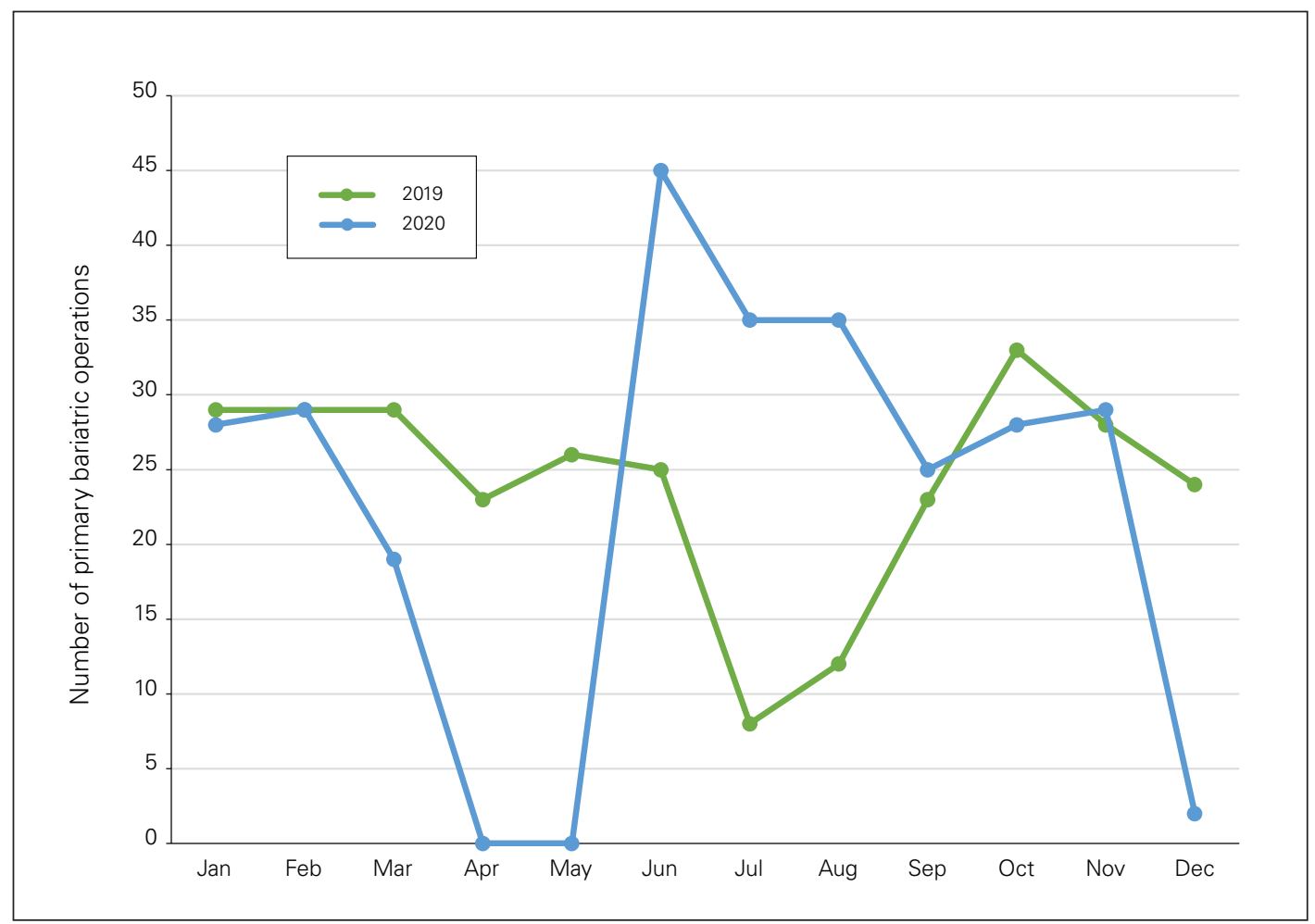

Fig. 1. Comparison of bariatric surgeries during the pandemic and the year prior, showing approximately a $79 \%$ reduction in the number of bariatric surgeries during the pandemic slow-down months (March, April, May and December 2020). 
course following Roux-en-Y gastric bypass (RYGB) or sleeve gastrectomy (SG), which was a fundamental component of reducing the burden on the health care system. A future addition to these measures is same-day surgery for selected patients undergoing uncomplicated RYGB or SG.

There are many more urgent surgeries that should precede bariatric surgeries, as delaying these procedures would lead to a rapid demise of the affected patients. However, long-term delays in bariatric surgery remain economically disadvantageous, as shown by its beneficial effects on morbidity and mortality, ${ }^{1,2}$ such as the resolution of diabetes and hypertension, and the improvement of dyslipidemia and nonalcoholic fatty liver disease, which can be seen as early as 1 month to 1 year after sugery, respectively.,5 Furthermore, patients scheduled for bariatric surgery often follow a strict, low-calorie liquid diet to ensure adequate preoperative weight loss and a reduction in liver size. Thus, delays in surgery can be detrimental to the mental and physical health of patients awaiting surgery, which can lead to patient frustration and noncompliance with the bariatric programs. Nonetheless, choosing the optimal bariatric patients - patients who are younger than 65 years, have a body mass index below $45 \mathrm{~kg} / \mathrm{m}^{2}$, are ambulatory and do not suffer from cardiac or respiratory comorbidities ${ }^{2}-$ is necessary to reduce the risk of adverse surgical events and potential admissions to the intensive care unit (ICU), while still allowing essential operations to proceed.

\section{Conclusion}

The COVID-19 pandemic has affected health care systems worldwide. Nevertheless, an abundance of knowledge has been gained from the pandemic. Obesity and its related diseases are associated with a higher risk of death from COVID-19. Consequently, delaying bariatric operations during the pandemic for a prolonged period might lead to further long-term strain on health care systems due to the morbidity associated with obesity. We advocate that classifying bariatric surgeries as nonelective and time-sensitive is essential. Using a standardized and specific ERAS bariatric protocol, which allows for most patients to be discharged on postoperative day 1 , is a potential solution to the issue of reduced resources and bed availability. Finally, choosing the optimal bariatric patient is crucial to preventing postoperative morbidity and ICU admissions during the pandemic. We believe that applying these simple but effective solutions in the context of a possible prolonged COVID19 pandemic will facilitate the sustained delivery of bariatric surgery to our population, which is significantly affected by obesity and its complications.

Affiliations: From the Department of Surgery, University of Alberta, Edmonton, Alta.

Competing interests: None declared.

Contributors: All authors contributed substantially to the conception, writing and revision of this article and approved the final version for publication.

Content licence: This is an Open Access article distributed in accordance with the terms of the Creative Commons Attribution (CC BYNC-ND 4.0) licence, which permits use, distribution and reproduction in any medium, provided that the original publication is properly cited, the use is noncommercial (i.e., research or educational use), and no modifications or adaptations are made. See: https://creativecommons. org/licenses/by-nc-nd/4.0/

\section{References}

1. Popkin BM, Du S, Green WD, et al. Individuals with obesity and COVID-19: a global perspective on the epidemiology and biological relationships. Obes Rev 2020;21: e13128.

2. Kompaniyets L, Goodman AB, Belay B, et al. Body mass index and risk for COVID-19-related hospitalization, intensive care unit admission, invasive mechanical ventilation, and death - United States, March-December 2020. MMWR Morb Mortal Wkly Rep 2021;70:355-61.

3. Executive Council of ASMBS. Safer through surgery: American Society for Metabolic and Bariatric Surgery statement regarding metabolic and bariatric surgery during the COVID-19 pandemic. Surg Obes Relat Dis 2020;16:981-82.

4. Małczak P, Pisarska M, Piotr M, et al. Enhanced recovery after bariatric surgery: systematic review and meta-analysis. Obes Surg 2016;27:226-35.

5. Scopinaro N, Papadia F, Camerini G, et al. A comparison of a personal series of biliopancreatic diversion and literature data on gastric bypass help to explain the mechanisms of resolution of type 2 diabetes by the two operations. Obes Surg 2008;18:1035-8. 